\title{
PTEN loss and level of HER2 amplification is associated with trastuzumab resistance and prognosis in HER2-positive gastric cancer
}

\author{
Chan Kim ${ }^{1, *}$, Choong-Kun Lee ${ }^{2, *}$, Hong Jae Chon ${ }^{1, *}$, Joo Hoon Kim ${ }^{2}$, Hyung Soon \\ Park ${ }^{2}$, Su Jin $\mathrm{Heo}^{2}$, Hyun Jeong Kim³, Tae Soo Kim³, Woo Sun Kwon ${ }^{3}$, Hyun Cheol \\ Chung ${ }^{2,3,4}$ and Sun Young Rha ${ }^{2,3,4}$ \\ ${ }^{1}$ Medical Oncology, CHA Bundang Medical Center, CHA University, Seongnam, Korea \\ ${ }^{2}$ Yonsei Cancer Center, Yonsei University College of Medicine, Seoul, Korea \\ ${ }^{3}$ Song Dang Institute for Cancer Research, Seoul, Korea \\ ${ }^{4}$ Brain Korea 21 PLUS Project for Medical Science, Yonsei University College of Medicine, Seoul, Korea \\ "These authors contributed equally to this study \\ Correspondence to: Sun Young Rha, email: rha7655@yuhs.ac \\ Keywords: trastuzumab; resistance; gastric cancer; HER2; PTEN \\ Received: August 08, $2017 \quad$ Accepted: November 13, $2017 \quad$ Published: December 09, 2017 \\ Copyright: Kim et al. This is an open-access article distributed under the terms of the Creative Commons Attribution License 3.0 \\ ( $C$ C BY 3.0), which permits unrestricted use, distribution, and reproduction in any medium, provided the original author and source \\ are credited.
}

\section{ABSTRACT}

Background: Trastuzumab is an active agent against human epidermal growth factor receptor 2 (HER2)-positive gastric cancer (GC). This study aimed to characterize resistance to trastuzumab-based front-line chemotherapy in HER2+ GC patients and to establish factors predictive of this resistance.

Results: Among 129 HER2+ GC patients, 25\% displayed rapid disease progression within 4 months from initiation of therapy. These patients showed a higher rate of signet ring cell histology, bone metastasis, poor performance status, frequent loss of PTEN expression, and low HER2 amplification index compared with patients who were progression-free for at least 4 months. In contrast, there was no significant difference in the frequency of the PIK3R1 variant. Multivariate analyses confirmed two independent molecular predictors for trastuzumab resistance: loss of PTEN expression and low HER2 amplification index $(<5)$. Patients with one or both molecular predictors at diagnosis exhibited worse progression-free and overall survival compared to those without risk factors $(p<0.001$ and $p=0.001$, respectively).

Conclusion: In HER2+ GC patients, loss of PTEN expression and low HER2 AI correlated with resistance to trastuzumab-based therapy and dismal prognosis. Since patients harboring these molecular predictors are unlikely to respond to trastuzumab-based therapy, other novel therapeutic targets needed to be considered.

Methods: HER2+ GC patients who were treated with trastuzumab in combination with either 5-fluorouracil/cisplatin or capecitabine/cisplatin were enrolled. Clinicopathologic features and molecular alterations of HER2, phosphoinositide 3-kinase regulatory subunit 1 (PIK3R1), and phosphatase and tensin homolog (PTEN) were correlated with treatment outcome. Factors predictive of resistance were also explored. 


\section{INTRODUCTION}

Trastuzumab (Herceptin, Roche) is a potent antiHER2 humanized monoclonal antibody directed against HER2+ gastric cancer (GC) $[1,2]$. The pivotal ToGA (Trastuzumab for Gastric Cancer) trial showed that addition of trastuzumab to conventional chemotherapy significantly prolongs overall survival (OS) and progression-free survival (PFS) in HER2+ GC compared with chemotherapy alone [3]. Trastuzumab-based regimen is now being considered as a standard front-line treatment for patients with HER2+ GC, and the median OS of these patients has risen to $>12$ months [3-5]. However, a proportion of HER2+ GC patients do not respond to trastuzumab-containing front-line chemotherapy ab initio, progressing rapidly within 3-4 months, and displaying dismal prognosis despite treatment $[3,6]$. These patients with refractory malignancies seem to have primary (de novo) resistance and currently, detailed characterization of these patients and availability of surrogate marker to predict primary resistance remain unknown.

Activation of the PI3K signaling pathway is common in various human malignancies. Moreover, the deregulation of PI3K subunits and PTEN are well-known to be involved during carcinogenesis [7-9]. Specifically, a weakened response to trastuzumab therapy was often seen in HER2+ breast cancer containing activating mutations of the PIK3CA gene and deletions of PTEN $[10,11]$. It has also been found that PTEN is not only a tumor suppressor, but also plays a critical role in trastuzumab susceptibility of HER2-amplified breast cancer cell lines [12]. Recently, PIK3R1, the regulatory subunit of PI3K, was also known to be involved in the activation of PI3K pathway and cancer progression [7, 13]. Genetic aberrations of PIK3R1 was reported in various tumors [13-16] and, intriguingly, tumors lacking PIK3CA mutations tended to have frequent mutations in PIK3R1 [15]. PIK3R1 mutant protein is known to bind and stabilize PTEN, thereby suppressing PTEN degradation in tumor cell [13]. In a previous study [17], we were unable to confirm the predictive role of PIK3CA in HER2+ GC; therefore, here we aimed to identify the role of other regulators of the PI3K signaling pathway in HER2+ GC: PTEN and PIK3R1.

In this study, we aimed to characterize HER2+ GC patients with resistance to trastuzumab-based therapy by focusing on the PI3K pathway, in order to establish molecular predictive factors for primary resistance.

\section{RESULTS}

\section{Baseline patient characteristics}

For 10 years, a total of 129 HER2+ GC patients were enrolled and treated with either trastuzumab/XP or trastuzumab/FP as a first-line chemotherapy. The clinicopathologic and molecular characteristics of HER2+ GC patients are summarized in Table 1. The median age at diagnosis was 59 years (range: $30-84$ ) and the maleto-female ratio was 2.9:1. Two-thirds of patients initially presented with distant metastases, whereas cancer recurred after previous curative gastrectomy in the remaining third. The most frequent metastatic site was the liver $(41.1 \%)$, followed by peritoneum (34.9\%), lung (12.4\%) and bone (11.6\%).

\section{HER2, PTEN, and PIK3R1 status and their association with patient characteristics}

HER2 expression in IHC staining was $3+$ in 98 (76.0\%) patients and $2+$ in the remaining $31(24.0 \%)$ patients. The mean amplification index (AI) of HER2 expression determined by FISH analysis was 7.0. The median follow-up duration was 23.8 months (range 0.961.8). We observed intact PTEN expression in $83.6 \%$ of GC tissues and loss of PTEN expression in $16.4 \%$ of GC tissues. The PIK3R1 wildtype allele (GG) was identified in $76.8 \%$ of patients, while the remaining $23.2 \%$ had a variant allele (GA or AA).

In 129 trastuzumab-treated patients, median PFS was 7.4 months and median OS was 16.0 months. Because there is no consensus on the criteria for dividing GC patients into trastuzumab-sensitive or -resistant, the lower quartile value of PFS (4.0 months) was used as a cutoff value to distinguish between patients who benefitted clinically from trastuzumab and those who did not. As a result, 32 patients $(25 \%)$ with PFS shorter than 4 months were classified as resistant and 97 patients $(75 \%)$ with more than 4 months, as sensitive.

The association between clinicopathological and molecular features in trastuzumab-resistant patients are also summarized in Table 1. In HER2+ GC patients with primary resistance, increased signet ring cell (SRC) histology $(21.9 \%$ vs. $6.2 \%$, $p=0.031)$ and a higher frequency of initial bone metastasis $(25.0 \%$ vs. $7.2 \%, p<0.025)$ was observed along with poor performance status (ECOG $\geq 2$ : $18.7 \%$ vs. $9.2 \%, p=0.001)$. Notably, the existence of initial bone metastasis was highly associated with poor performance status $(p=0.002)$, suggesting that bone metastasis in GC severely impairs the patient's activities of daily living. The average AI of HER2 in FISH analysis was significantly lower in patients with primary resistance compared with sensitive patients ( $7.7 \pm 6.3$ vs. $4.1 \pm 1.4, p=0.005)$. Moreover, loss of PTEN expression was more common in trastuzumabresistant HER2 + GC patients than in those who were sensitive to treatment $(38.5 \%$ vs. $11.1 \%, p=0.017)$. However, there was no significant difference in the frequency of the PIK3R1 variant allele between patients with and without trastuzumab-resistance. 
Table 1: Clinicopathologic features of HER2+ gastric cancer

\begin{tabular}{|c|c|c|c|c|}
\hline & All patients $(n=129)$ & $\begin{array}{c}\text { Trastuzumab-resistant } \\
\text { patient }(n=32)\end{array}$ & $\begin{array}{c}\text { Trastuzumab-sensitive } \\
\text { patients }(n=97)\end{array}$ & $P$-value \\
\hline Age at diagnosis & $59(30-84)$ & $60(30-82)$ & $58(32-84)$ & 0.623 \\
\hline Sex & & & & 0.931 \\
\hline Male & $96(74.4 \%)$ & $24(75.0 \%)$ & $72(74.2 \%)$ & \\
\hline Female & $33(25.6 \%)$ & $8(25.0 \%)$ & $25(25.8 \%)$ & \\
\hline Histology & & & & 0.031 \\
\hline Adeno, WD & $7(5.4 \%)$ & $0(0 \%)$ & $7(7.2 \%)$ & \\
\hline Adeno, MD & $76(58.9 \%)$ & $16(50.0 \%)$ & $60(61.9 \%)$ & \\
\hline Adeno, PD & $33(25.6 \%)$ & $9(28.1 \%)$ & $24(24.7 \%)$ & \\
\hline SRC carcinoma & $13(10.1 \%)$ & $7(21.9 \%)$ & $6(6.2 \%)$ & \\
\hline Previous surgery & & & & 0.491 \\
\hline Yes & $42(32.6 \%)$ & $12(37.5 \%)$ & $30(30.9 \%)$ & \\
\hline No & $87(67.4 \%)$ & $20(62.5 \%)$ & $67(69.1 \%)$ & \\
\hline Location of tumor & & & & 0.420 \\
\hline Proximal & $31(24.0 \%)$ & $6(18.8 \%)$ & $25(25.8 \%)$ & \\
\hline Distal & $98(76.0 \%)$ & $26(81.3 \%)$ & $72(74.2 \%)$ & \\
\hline Peritoneal metastasis & & & & 0.225 \\
\hline Yes & $45(34.9 \%)$ & $14(43.8 \%)$ & $31(32.0 \%)$ & \\
\hline No & $84(65.1 \%)$ & $18(56.3 \%)$ & $66(68.0 \%)$ & \\
\hline Liver metastasis & & & & 0.951 \\
\hline Yes & $53(41.1 \%)$ & $13(40.6 \%)$ & $40(41.2 \%)$ & \\
\hline No & $76(58.9 \%)$ & $19(59.4 \%)$ & $57(58.8 \%)$ & \\
\hline Bone metastasis & & & & 0.025 \\
\hline Yes & $15(11.6 \%)$ & $8(25.0 \%)$ & $7(7.2 \%)$ & \\
\hline No & $104(80.6 \%)$ & $22(68.8 \%)$ & $82(84.5 \%)$ & \\
\hline N/A & $10(7.8 \%)$ & $2(6.3 \%)$ & $8(8.2 \%)$ & \\
\hline Lung metastasis & & & & 0.985 \\
\hline Yes & $16(12.4 \%)$ & $4(12.5 \%)$ & $12(12.4 \%)$ & \\
\hline No & $113(87.6 \%)$ & $28(87.5 \%)$ & $85(87.6 \%)$ & \\
\hline Performance status & & & & 0.001 \\
\hline ECOG 0 & $72(55.8 \%)$ & $8(25.0 \%)$ & $64(66.0 \%)$ & \\
\hline ECOG 1 & $42(32.6 \%)$ & $18(56.3 \%)$ & $24(24.7 \%)$ & \\
\hline $\mathrm{ECOG} \geq 2$ & $15(11.7 \%)$ & $6(18.7 \%)$ & $9(9.2 \%)$ & \\
\hline Regimen & & & & 0.896 \\
\hline Herceptin-FP & $17(13.2 \%)$ & $4(12.5 \%)$ & $13(13.4 \%)$ & \\
\hline Herceptin-XP & $112(86.8 \%)$ & $28(87.5 \%)$ & $84(86.6 \%)$ & \\
\hline HER2 IHC & & & & 0.475 \\
\hline $2+$ & $31(24.0 \%)$ & $10(31.3 \%)$ & $21(21.6 \%)$ & \\
\hline $3+$ & $98(76.0 \%)$ & $22(68.8 \%)$ & $76(78.4 \%)$ & \\
\hline HER2 amplification index & $7.0 \pm 5.9$ & $7.7 \pm 6.3$ & $4.1 \pm 1.4$ & 0.005 \\
\hline PTEN expression & & & & 0.017 \\
\hline Loss & $11(16.4 \%)$ & $5(38.5 \%)$ & $6(11.1 \%)$ & \\
\hline Intact & $56(83.6 \%)$ & $8(61.5 \%)$ & $48(88.9 \%)$ & \\
\hline PIK3R1 genotype & & & & 0.460 \\
\hline Variant & $16(23.2 \%)$ & $5(31.3 \%)$ & $12(22.2 \%)$ & \\
\hline Wildtype & $53(76.8 \%)$ & $11(68.8 \%)$ & $42(77.8 \%)$ & \\
\hline
\end{tabular}


Table 2: Clinical response to trastuzumab-based first-line chemotherapy

\begin{tabular}{|c|c|c|c|c|}
\hline & \multicolumn{2}{|c|}{ HER2 AI } & \multicolumn{2}{|c|}{ PTEN expression } \\
\hline & $\geq 5$ & $<5$ & Intact & Loss \\
\hline \multicolumn{5}{|c|}{ Best response } \\
\hline CR, PR & $47(58.8 \%)$ & $6(21.4 \%)$ & $36(64.3 \%)$ & $4(36.4 \%)$ \\
\hline SD & $26(32.5 \%)$ & $15(53.6 \%)$ & $15(26.8 \%)$ & $5(45.5 \%)$ \\
\hline PD & $7(8.8 \%)$ & $7(25.0 \%)$ & $5(8.9 \%)$ & $2(18.2 \%)$ \\
\hline$P$-value & \multicolumn{2}{|c|}{0.002} & \multicolumn{2}{|c|}{0.220} \\
\hline
\end{tabular}

Table 3: Univariate and multivariate analysis for PFS and OS

\begin{tabular}{|c|c|c|c|c|}
\hline & \multicolumn{2}{|c|}{ Univariate } & \multicolumn{2}{|c|}{ Multivariate } \\
\hline & $\begin{array}{c}\text { Median survival } \\
\text { (months) }\end{array}$ & $P$-value & HR $(95 \%$ CI $)$ & $P$-value \\
\hline \multicolumn{5}{|l|}{ PFS } \\
\hline Histology (SRC vs. non-SRC) & 5.1 vs. 8.2 & 0.103 & $1.27(0.46-3.49)$ & 0.647 \\
\hline Bone metastasis (Yes vs. No) & 2.8 vs. 8.2 & 0.001 & $1.57(0.57-4.33)$ & 0.383 \\
\hline ECOG Performance ( $\geq 1$ vs. 0 ) & 5.1 vs. 12.1 & $<0.001$ & $1.14(0.52-2.49)$ & 0.742 \\
\hline HER2 AI (<5vs. $\geq 5)$ & 4.0 vs. 9.5 & $<0.001$ & $3.26(1.38-7.73)$ & 0.007 \\
\hline PTEN expression (loss vs. intact) & 4.5 vs. 12.4 & 0.004 & $3.01(1.22-7.46)$ & 0.017 \\
\hline \multicolumn{5}{|l|}{$\mathbf{O S}$} \\
\hline Histology (SRC vs. non-SRC) & 6.5 vs. 16.4 & 0.010 & $1.58(0.43-5.76)$ & 0.491 \\
\hline Bone metastasis (Yes vs. No) & 14.6 vs. 16.9 & 0.009 & $2.21(0.70-7.02)$ & 0.179 \\
\hline ECOG Performance ( $\geq 1$ vs. 0 ) & 9.8 vs. 23.5 & $<0.001$ & $1.01(0.37-2.74)$ & 0.987 \\
\hline HER2 AI (<5vs. $\geq 5)$ & 11.1 vs. 20.8 & 0.001 & $3.23(1.23-8.49)$ & 0.018 \\
\hline PTEN expression (loss vs. intact) & 12.3 vs. 28.9 & 0.011 & $2.88(1.02-8.10)$ & 0.045 \\
\hline
\end{tabular}

\section{HER2 and PTEN status and clinical response to trastuzumab-based therapy}

Next, we analyzed clinical outcomes according to the molecular characteristics of HER2+ GC. In terms of tumor response to first-line therapy (Table 2), objective response rate was significantly increased in patients with HER2 AI $\geq 5$ compared to those with AI $<5$ (58.8\% vs. $21.4 \%, p=0.002)$. At the same time, objective response rate tended to decrease in patients with a loss of PTEN compared to those with intact PTEN expression $(64.3 \%$ vs. $36.4 \%, p=0.220)$.

\section{Low HER2 amplification index and loss of PTEN expression predict resistance to trastuzumab- based first-line chemotherapy}

To identify the factors predictive of response to trastuzumab-based first-line therapy, we performed univariate analyses with various clinicopathologic and molecular characteristics of first-line PFS and OS (Table 3). In univariate analysis, SRC histology, initial presence of bone metastasis, poor performance status, HER2 AI $<5$, and loss of PTEN expression were predictive for shorter
PFS in HER2+ GC patients when treated with Herceptin. Next, to verify independent predictive factors for primary resistance, we performed multivariate analyses for variables found to be statistically significant in univariate analyses. Multivariate Cox regression analysis revealed two independent predictors for primary resistance against trastuzumab-based regimen: low HER2 AI $(<5)$ and loss of PTEN expression. In patients with HER2 AI $<5$, median PFS and OS of first-line therapy was lower compared to those with HER $2 \mathrm{AI} \geq 5$ (PFS: 4.0 vs. 9.5 months, $p<0.001$; OS: 11.1 vs. 20.8 months, $p=0.001$ ) (Figure 1A). Moreover, in patients with a loss of PTEN expression, median PFS and OS of first-line trastuzumab-based treatment was shortened compared to those with intact PTEN expression (PFS: 4.5 vs. 12.4 months, $p=0.004$; OS: 12.3 vs. 28.9 months, $p=0.011$ ) (Figure 1B).

Next, we analyzed PFS and OS according to the number of these risk factors that patients possessed: 0 vs. 1 vs. 2. (Figure 1C). The most favorable survival outcome was seen in patients with no risk factors: median PFS was 9.0 months (95\% CI: 6.8-11.2) and OS 20.8 months (95\% CI: 12.8-28.7). The prognosis of patients possessing 1 risk factor was intermediate with a median PFS of 5.8 months (95\% CI: 6.8-11.2) and OS of 15.8 months 
(95\% CI: 9.4-22.1). However, the patients with both risk factors had the worst prognosis with a median PFS of 2.6 months (95\% CI: $2.1-3.1)$ and OS of 4.8 months (95\% CI: 0-9.9). Based on these findings, we could confirm that these two molecular factors are the main predictors of primary resistance to trastuzumab in HER2+ GC patients.

\section{DISCUSSION}

Trastuzumab is an active agent used in combination with chemotherapy against HER2+ metastatic GC [1] Since its approval by the FDA in 2010, it is widely



used in the treatment of metastatic HER2+ GC. In Eastern countries, the majority of patients treated with trastuzumab-based chemotherapy presented with PFS > 6 months and OS $>12$ months $[17,18]$. However, some HER2+ GC patients exhibited primary resistance to trastuzumab-based regimen. These patients progressed rapidly within a few months of initiation of therapy, and eventually displayed dismal prognosis [6].

To date, HER2+ GC patients with primary resistance to trastuzumab have not been thoroughly characterized, and only a few studies on the predictive biomarkers for trastuzumab resistance in GC have been
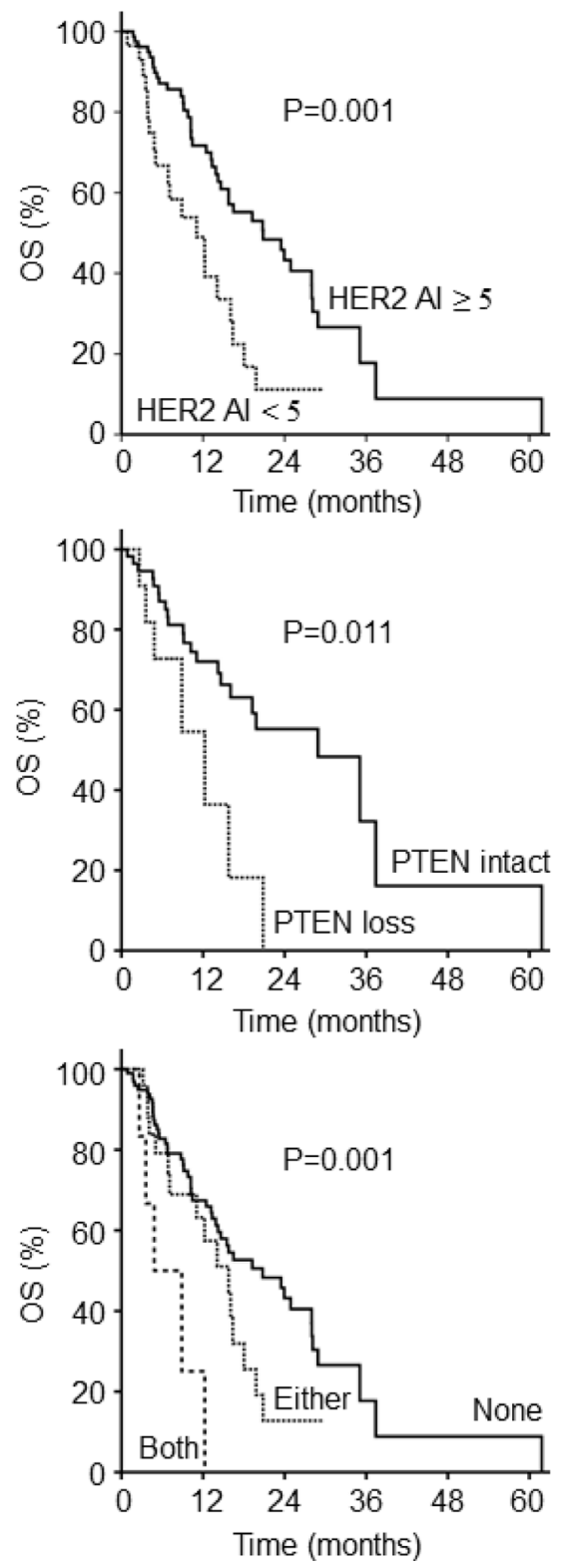

Figure 1: Survival outcomes according to molecular biomarkers in HER2+ gastric cancer. (A) PFS and OS according to the level of HER2 amplification (B) PFS and OS according to the presence or absence of PTEN expression (C) PFS and OS according to the number of molecular biomarker PFS, progression-free survival; OS, overall survival. 
reported. In the post-hoc analysis of the ToGA trial, a link was reported between the baseline HER2 expression level and trastuzumab response [3, 18]. In addition, Gomez-Martin et al. recently suggested that the level of HER2 amplification predicts treatment response and overall survival in GC [19]. In most recent study, Deguchi et al. revealed that the knockdown of PTEN induces trastuzumab resistance by Akt activation in preclinical model of GC [20]. They also reported that there is no clinical response in PTEN-deficient GC patients compared to response rate of $50 \%$ in PTENpositive GC patients. However, since the number of HER $2+$ patients in this study was very small $(n=23)$, the results should be interpreted with care. Moreover, there are reports on the relationship between PTEN expression and response to the trastuzumab-containing therapy in HER2+ breast cancer [21]. In summary, comprehensive studies on the potential biomarkers of HER2+ GC, whether they be clinical or molecular, are still lacking and need to be pursued.

In the present study, we found that trastuzumabresistant HER2 + GC patients usually presented with SRC histology, initial bone metastasis, poor performance status $($ ECOG $\geq 2)$, low HER2 AI in FISH and a loss of PTEN expression at diagnosis. Although we also examined the role of PIK3R1, the regulatory subunit of PI3K, we were unable to determine its role in the treatment of HER2+ GC. Moreover, following multivariate Cox regression analysis, we were able to identify two independent molecular predictors for resistance to first-line trastuzumab-containing chemotherapy: low HER2 AI $(<5)$ and loss of PTEN expression. These two risk factors are also independent prognostic factors for OS. Patients possessing either of these risk factors exhibited primary resistance to trastuzumab and their prognosis is dismal, suggesting that this subgroup would benefit from other therapeutic targets. Because loss of PTEN expression is associated with the activation of PI3K signaling and mitogen-activated protein kinase (MAPK) pathways [22], pharmacologic inhibition of these pathways could provide a reasonable strategy to overcome trastuzumab resistance in HER2+ GC.

In this study, patients with SRC histology responded poorly to trastuzumab-based regimen. Previous research has shown that epithelial-to-mesenchymal transition (EMT) confers primary resistance to trastuzumab in tumor cells [23]. In addition, the loss of E-cadherin in gastric mucosal epithelial cells is a critical carcinogenic event that initiates gastric SRC carcinoma in humans and mice [24]. Therefore, primary resistance in SRC carcinoma may be the result of augmented EMT in E-cadherin-deficient $\mathrm{SRC}$. More preclinical evidence is required to prove this inference.

The findings of this study may be limited by its retrospective nature. However, we believe that these shortcomings can be offset to a certain extent by the fact that more than half of the patients in this study formed part of well-controlled, multi-institutional prospective clinical trials such as the ToGA trial, and that the remaining patients received standardized clinical treatment at a single tertiary cancer center. To validate the findings of the present study and further characterize the biology of HER2 $+\mathrm{GC}$, we are now planning to establish the multicenter prospective cohort for HER2+ GC patients in Korea.

In conclusion, loss of PTEN expression and low HER2 AI correlated with resistance to trastuzumab-based first-line therapy and dismal prognosis in HER2+ GC. Since patients harboring these molecular alterations are unlikely to respond to conventional trastuzumab-based therapy, other novel therapeutics that will benefit this subset need to be pursued.

\section{MATERIALS AND METHODS}

\section{Patient selection}

Patients with metastatic GC were enrolled in Yonsei Cancer Center, Severance Hospital, Seoul, Korea between December 2005 and August 2015. Patients were followed up until December 2016. Patient eligibility criteria were as follows: 1) metastatic GC patients with HER2 positivity defined by either HER2 $3+$ in immunohistochemical (IHC) staining, or HER2 2+ in IHC staining and HER2 amplification on fluorescence in situ hybridization (FISH) (HER2:CEP17 ratio $\geq 2$ ); 2) chemotherapy-naive patients with the exception of adjuvant chemotherapy 6 months prior to enrollment; 3) patients who have been treated with palliative trastuzumab in combination with either 5-fluorouracil and cisplatin (FP) or capecitabine and cisplatin (XP). Clinicopathologic parameters were reviewed from the information of the electronic medical record system as previously described [25].

HER2 status in surgical or biopsy specimen were analyzed and determined by experienced pathologists in Severance Hospital, Seoul, Korea, using the HercepTest $\mathrm{Kit}^{\mathrm{TM}}$ (DAKO, Denmark) for IHC staining and Vysis ${ }^{\mathrm{TM}}$ HER2/CEP FISH probe kit (Abbott, USA) for FISH analysis according to the manufacturers' instructions. This study was approved by the Institutional Review Board in Severance Hospital, Seoul, Korea (IRB approval number: 4-2014-1076).

\section{Evaluation of PTEN expression in GC tissue}

Formalin-fixed paraffin-embedded sections of tumor tissue were deparaffinized with xylene and hydrated with graded alcohol. Antigen retrieval was performed with a retrieval solution (DAKO, USA) using the pressure-cooking method, and the activity of endogenous peroxidase was blocked by a 1:40 mixture of hydrogen peroxide and methanol. The primary PTEN antibody (DAKO) incubation was performed at room 
temperature for $1 \mathrm{~h}$ in an antibody solution diluted to 1:100. All sections were incubated at room temperature for $30 \mathrm{~min}$ in the Real EnVision ${ }^{\mathrm{TM}}$ HRP Rabbit/Mouse (DAKO, USA) detection system, which functions as the secondary antibody. PTEN expression was indicated using a chromogen and counterstaining was performed with hematoxylin. PTEN expression was quantified by the H-score based on the intensity of cell staining and percentage of the stained cells [17]. Intensity was scored as 0 : none, 1: weak, 2: moderate, or 3: strong. The H-score was calculated as follows: $\mathrm{H}$-score $=(\% 1+$ cells $\times 1)$ $+(\% 2+$ cells $\times 2)+(\% 3+$ cells $\times 3)$. An H-score of $\leq$ 10 was used as the cutoff point to define loss of PTEN expression based on a previous study.

\section{PIK3R1 variant analysis}

We used a pyrosequencing assay to detect variant alleles of PIK3R1. The primer was forward 5' (biotin) CACCAAAACCTACTACTGTAGCCAA-3' and reverse 5' - GAGATATCTCCCCAGTACCATTCA-3'. Amplicon length was $85 \mathrm{bp}$ and sequence to analyze was 5'-AGGA C/T ATATT-3'. Each PCR mix contained the forward and reverse primers, dNTP mix, $\mathrm{MgCl}_{2}$, PCR buffer, AmpliTaq Gold, and $100 \mathrm{ng}$ of sample genomic DNA. PCR mixtures were denatured for $10 \mathrm{~min}$ at $95^{\circ} \mathrm{C}$ and then thermalcycled for $30 \mathrm{~s}$ at $95^{\circ} \mathrm{C}, 30 \mathrm{~s}$ at $55^{\circ} \mathrm{C}$, and $30 \mathrm{~s}$ at $72^{\circ} \mathrm{C}$, repeating the cycle 40 times. A final extension step at $72^{\circ} \mathrm{C}$ for $5 \mathrm{~min}$ completed the program. PCR products were analyzed by agarose gel electrophoresis and sequenced using the PyroMark Q24 (QIAGEN) system according to the manufacturer's instructions.

\section{Statistical analysis}

The correlation between clinicopathologic variables was compared using chi-square test and independent sample $t$-test. Multivariate analysis was done by logistic regression to identify the independent predictive factors for primary resistance to trastuzumab. OS was defined as the time interval from the date of initiation of trastuzumabbased chemotherapy to the date of death or last followup. PFS was defined as the time between initiation of therapy and the date of documented disease progression or death. All patients underwent response evaluation with regular CT scan at 6- to 8-week intervals for accurate PFS measurement. OS and PFS were compared using KaplanMeier survival analyses with log-rank tests. The accepted level of statistical significance was $p<0.05$. All statistical analyses were carried out with SPSS 12.0 (SPSS, Inc.).

\section{ACKNOWLEDGMENTS AND FUNDING}

This research was supported by a grant of the Korea Health Technology R\&D Project through the Korea Health Industry Development Institute (KHIDI), funded by the Ministry of Health \& Welfare, Republic of Korea (HI13C2096 to S.Y.R.) and by the National Research Foundation of Korea (NRF) funded by the Ministry of Science, ICT \& Future Planning (NRF-2017R1A2B2005772 to S.Y.R. and NRF-2016R1C1B2014671 to C.K.).

\section{CONFLICTS OF INTEREST}

All authors disclosed no potential conflicts of interest.

\section{Human rights and informed consent}

All procedures followed were in accordance with the ethical standards of the responsible committee on human experimentation (institutional and national) and with the Helsinki Declaration of 1964 and later versions. Informed consent or substitute for it was obtained from all patients for being included in the study.

\section{REFERENCES}

1. Gomez-Martín C, Lopez-Rios F, Aparicio J, Barriuso J, García-Carbonero R, Pazo R, Rivera F, Salgado M, Salud A, Vázquez-Sequeiros E. A critical review of HER2-positive gastric cancer evaluation and treatment: from trastuzumab, and beyond. Cancer Lett. 2014; 351:30-40.

2. Boku N. HER2-positive gastric cancer. Gastric Cancer. 2014; 17:1-12.

3. Bang YJ, Van Cutsem E, Feyereislova A, Chung HC, Shen L, Sawaki A, Lordick F, Ohtsu A, Omuro Y, Satoh T. Trastuzumab in combination with chemotherapy versus chemotherapy alone for treatment of HER2-positive advanced gastric or gastro-oesophageal junction cancer (ToGA): a phase 3, open-label, randomised controlled trial. The Lancet. 2010; 376:687-697.

4. Gomez-Martín C, Lopez-Rios F, Aparicio J, Barriuso J, García-Carbonero R, Pazo R, Rivera F, Salgado M, Salud A, Vázquez-Sequeiros E. A critical review of HER2-positive gastric cancer evaluation and treatment: From trastuzumab, and beyond. Cancer Lett. 2014.

5. Park SC, Chun HJ. Chemotherapy for advanced gastric cancer: review and update of current practices. Gut Liver. 2013; 7(4):385.

6. Cid RAP, Antón A. Advanced HER2-positive gastric cancer: current and future targeted therapies. Crit Rev Oncol Hematol. 2013; 85:350-362.

7. Fruman DA, Rommel C. PI3K and cancer: lessons, challenges and opportunities. Nature reviews Drug discovery. 2014; 13:140-156.

8. Martini M, De Santis MC, Braccini L, Gulluni F, Hirsch E. PI3K/AKT signaling pathway and cancer: an updated review. Ann Med. 2014; 46:372-383. 
9. Mayer IA, Arteaga CL. The PI3K/AKT pathway as a target for cancer treatment. Annu Rev Med. 2016; 67:11-28.

10. Esteva FJ, Guo H, Zhang S, Santa-Maria C, Stone S, Lanchbury JS, Sahin AA, Hortobagyi GN, Yu D. PTEN, PIK3CA, p-AKT, and p-p70S6K status: association with trastuzumab response and survival in patients with HER2positive metastatic breast cancer. Am J Pathol. 2010; 177:1647-1656.

11. Razis E, Bobos M, Kotoula V, Eleftheraki A, Kalofonos H, Pavlakis K, Papakostas P, Aravantinos G, Rigakos G, Efstratiou I. Evaluation of the association of PIK3CA mutations and PTEN loss with efficacy of trastuzumab therapy in metastatic breast cancer. Breast Cancer Res Treat. 2011; 128:447-456.

12. Nagata Y, Lan KH, Zhou X, Tan M, Esteva FJ, Sahin AA, Klos KS, Li P, Monia BP, Nguyen NT. PTEN activation contributes to tumor inhibition by trastuzumab, and loss of PTEN predicts trastuzumab resistance in patients. Cancer cell. 2004; 6:117-127.

13. Cheung LW, Hennessy BT, Li J, Yu S, Myers AP, Djordjevic B, Lu Y, Stemke-Hale K, Dyer MD, Zhang F. High frequency of PIK3R1 and PIK3R2 mutations in endometrial cancer elucidates a novel mechanism for regulation of PTEN protein stability. Cancer Discov. 2011:CD-11-0039.

14. Urick ME, Rudd ML, Godwin AK, Sgroi D, Merino M, Bell DW. PIK3R1 (p85 $\alpha$ ) is somatically mutated at high frequency in primary endometrial cancer. Cancer Res. 2011; 71:4061-4067.

15. Kandoth C, McLellan MD, Vandin F, Ye K, Niu B, Lu C, Xie M, Zhang Q, McMichael JF, Wyczalkowski MA. Mutational landscape and significance across 12 major cancer types. Nature. 2013; 502:333.

16. Kim C, Kwon WS, Rha SY, Kang SK, Kim H, BuserDoepner C, Yan L, Kumar R, Chung HC. Whole-exome sequencing of gastric cancer identifies germline PIK3R1 variant as a novel genetic biomarker for a PI3K betaisoform selective inhibitor, GSK2636771. AACR; 2015.

17. Zhang X, Park JS, Park KH, Kim KH, Jung M, Chung HC, Rha SY, Kim HS. PTEN deficiency as a predictive biomarker of resistance to HER2-targeted therapy in advanced gastric cancer. Oncology. 2015; 88:76-85.
18. Sawaki A, Ohashi Y, Omuro Y, Satoh T, Hamamoto Y, Boku N, Miyata Y, Takiuchi H, Yamaguchi K, Sasaki Y. Efficacy of trastuzumab in Japanese patients with HER2-positive advanced gastric or gastroesophageal junction cancer: a subgroup analysis of the Trastuzumab for Gastric Cancer (ToGA) study. Gastric Cancer. 2012; 15:313-322.

19. Gomez Martin C, Plaza JC, Pazo Cid R, Salud A, Pons F, Fonseca P, Leon A, Alsina M, Visa L, Rivera F. Level of HER2 gene amplification predicts response and overall survival in HER2-positive advanced gastric cancer treated with trastuzumab. J Clin Oncol. 2013; 31:4445-4452.

20. Deguchi Y, Okabe H, Oshima N, Hisamori S, Minamiguchi S, Muto M, Sakai Y. PTEN loss is associated with a poor response to trastuzumab in HER2-overexpressing gastroesophageal adenocarcinoma. Gastric Cancer. 2017; 20:416-427.

21. Gschwantler Kaulich D, Tan YY, Fuchs EM, Hudelist G, Köstler WJ, Reiner A, Leser C, Salama M, Attems J, Deutschmann C. PTEN expression as a predictor for the response to trastuzumab-based therapy in Her-2 overexpressing metastatic breast cancer. PloS one. 2017; 12:0172911.

22. Ebbesen SH, Scaltriti M, Bialucha CU, Morse N, Kastenhuber ER, Wen HY, Dow LE, Baselga J, Lowe SW. Pten loss promotes MAPK pathway dependency in HER2/neu breast carcinomas. Proceedings of the National Academy of Sciences. 2016; 113:3030-3035.

23. Oliveras Ferraros C, Corominas Faja B, Cufí S, Vazquez Martin A, Martin-Castillo B, Iglesias JM, López-Bonet E, Martin ÁG, Menendez JA. Epithelial-to-mesenchymal transition (EMT) confers primary resistance to trastuzumab (Herceptin). Cell Cycle. 2012; 11:4020-4032.

24. Humar B, Blair V, Charlton A, More H, Martin I, Guilford P. E-cadherin deficiency initiates gastric signet-ring cell carcinoma in mice and man. Cancer Res. 2009; 69:2050-2056.

25. Kim C, Yang H, Park I, Chon HJ, Kim JH, Kwon WS, Lee WS, Kim TS, Rha SY. Rho GTPase RhoJ is associated with gastric cancer progression and metastasis. J Cancer. 2016; 7:1550. 